\title{
Precision therapy for acute myeloid leukemia
}

\author{
Xue Yang and Jianxiang Wang
}

\begin{abstract}
Acute myeloid leukemia (AML) is a molecularly and clinically heterogeneous disease. Despite advances in understanding the pathogenesis of AML, the standard therapy remained nearly unchanged over the past three decades. With the poor survival for older patients and high relapse rate, multiple studies are ongoing to address this important issue. Novel therapies for AML, including the refinements of conventional cytotoxic chemotherapies and genetic and epigenetic targeted drugs, as well as immunotherapies, have been developed in recent years. Here, we present a mechanism-based review of some promising new drugs with clinical efficacy, focus on targeted drugs that are most potential to pave the road to success, and put forward the major challenges in promoting the precision therapy for AML.
\end{abstract}

Keywords: Acute myeloid leukemia, Precision therapy, Conventional chemotherapies, Molecular targeted inhibitors, Epigenetic mutations, Immunotherapy

\section{Background}

Acute myeloid leukemia (AML) represents a heterogeneous malignancy characterized by a clonal proliferation and impaired differentiation of myeloid precursors with diverse outcomes. Despite the advances in understanding the molecular heterogeneity and pathogenesis of AML, there has been little progress in the standard therapy for AML over the past four decades. The classic treatment ranges from cytarabine-based chemotherapy to hematopoietic stem cell transplantation (HSCT), with a 5-year overall survival (OS) of $40 \%$ for patients younger than 60 years. For those older than 60 years, who made up of the majority of AML cases, the 5-year OS was only 10 20\% [1, 2]. Few of patients who relapsed after complete remission (CR) could survive for more than 5 years [3].

Briefly, the treatment of AML consists mainly of remission induction and post-remission therapy which contains chemotherapy, targeted therapies, and HSCT. In terms of induction therapy, for adult patients with newly diagnosed AML, a combination of anthracycline for 3 days and standard-dose cytarabine for 7 to 10 days (" $7+3$ " therapy) are recommended. For elder patients

\footnotetext{
*Correspondence: wangjx@ihcams.ac.cn

State Key Laboratory of Experimental Hematology, Institute of Hematology and Blood Diseases Hospital, Chinese Academy of Medical Sciences and Peking Union Medical College, Tianjin, China
}

(> 60 years), the best chemotherapy remains to be identified. Most of them recommended the same remission induction regimen except those with unfavorable risk or severe commodity who are too fragile to tolerate intensive chemotherapy. When it comes to post-remission treatment including consolidation and maintenance therapy, risk stratification should be taken into consideration. AML patients are categorized based on cytogenetic, molecular, and clinical characteristics that are prognostic important. For younger patients, high-dose cytarabine are recommended in patients with favorable cytogenesis. While for those with adverse prognosis, allogeneic HSCT (allo-HSCT) should be performed in the first remission. For elder patients who fit for chemotherapy in the first complete remission (CR), consolidation therapy could contain anthracycline and cytarabine or intermediate-dose cytarabine alone. Likewise, those with unfavorable risk should be considered for nonmyeloablative HCT. Of note, for relapsed or refractory (R/R) AML population, allo-HSCT provides the highest likelihood of cure.

Among all AML subtypes, acute promyelocytic leukemia (APL) contributes the highest proportion of cure rate for patients undergoing targeted therapy such as alltransretinoic acid (ATRA) and arsenic trioxide, which implies a strong need for individualized medicine. With 
the advent in next-generation sequencing technologies, novel therapies have emerged, including multiple molecular target inhibitors and immunotherapies [Table 1].

\section{Heterogeneity of AML}

Tumor heterogeneity refers to distinct morphological and phenotypic features in tumors mainly arising from gene alterations, which has been observed in all types of tumors including leukemia. The heterogeneity of AML involves both genomic and epigenomic changes, including distinct sets of cytogenetic abnormalities and somatic mutations [4], resulting in a range of morphological, immunophenotypic, cytogenetic, biomolecular, and clinical features [5]. Moreover, in the course of the disease, the leukemic clone may change from diagnosis to relapse due to the heterogeneity of leukemia cells [6], and transformation of hematopoietic stem cells (HSCs)

Table 1 Examples of targeted drugs for AML

\begin{tabular}{|c|c|c|}
\hline Target & Drug & Phase of development \\
\hline PLKs & Volasertib & 3 \\
\hline \multirow[t]{6}{*}{ FLT3 } & Sorafenib & 2 \\
\hline & Midostaurin (PKC412) & 3 \\
\hline & Quizartinib (AC220) & 3 \\
\hline & Crenolanib (CP868596) & 2 \\
\hline & Gilteritinib (ASP2215) & 3 \\
\hline & Lestaurtinib (CEP-701) & 3 \\
\hline \multirow[t]{4}{*}{ DNMTs } & Azacitidine (5-Aza) & Approved \\
\hline & Decitabine & Approved \\
\hline & Guadecitabine (SGI-110) & 3 \\
\hline & Sapacitabine (CYC682) & 3 \\
\hline $\mathrm{IDH} 2$ & AG-221 & 3 \\
\hline $\mathrm{IDH} 1$ & AG-120 & 2 \\
\hline \multirow[t]{2}{*}{ HDACs } & Vorinostat & 3 \\
\hline & Entinostat & 2 \\
\hline BET & OTX015 & 1 \\
\hline DOT1L & Pinometostat (EPZ-2676) & 1 \\
\hline LSD1 & GSK2879552 & 1 \\
\hline \multirow[t]{3}{*}{ CD33 } & $\mathrm{GO}$ & 3 \\
\hline & SGN-33A & 3 \\
\hline & CD33 CART & Preclinical \\
\hline \multirow[t]{3}{*}{ CD123 } & CSL362 & Preclinical \\
\hline & SL-401 & Preclinical \\
\hline & CD123 CART & Preclinical \\
\hline PD-1 & Nivolumab & 2 \\
\hline CTLA4 & Ipilimumab & 2 \\
\hline
\end{tabular}

PLKs polo-like kinases, FLT3 Fms-like tyrosine kinase 3, DNMTs DNA methyltransferases, IDH isocitrate dehydrogenase, HDACs histone deacetylases, BET bromodomain and extra-terminal motif, DOT1L disruptor of telomeric silencing 1-like, $L S D 1$ lysine-specific histone demethylase $1 \mathrm{~A}, P D-1$ programmed cell death protein 1, CTLA4 cytotoxic T-lymphocyte-associated protein 4 to leukemia initiating cells occurs at different stages within primitive multipotent cells [7, 8]. Consequently, the heterogeneity of AML brings about varied response to treatment, as well as drug resistance and disease relapse, posing a challenge to personalized therapeutic regimens, likewise, known as precision medicine.

\section{Refinements of conventional cytotoxic chemotherapies}

Conventional intensive chemotherapy has a cure rate of only $30-50 \%$, and the majority of patients aged 70 years or older could not benefit from it due to poor tolerance and high mortality [9]. Despite $40-80 \%$ patients achieving $\mathrm{CR}$, the median survival in elderly patients receiving intensive chemotherapy is 4.6 months with a 1-year survival rate of only $28 \%$ [10]. Besides, a high frequency of subsequent relapse remains the major obstacle to overcome. In the past decades, studies have driven the improvements in OS by novel formulations and refinements of conventional chemotherapy.

\section{Intensification of the standard induction therapy}

The $7+3$ regimen, consisting of 7 days continuous infusion of cytarabine along with a short infusion or bolus of an anthracycline given on days 1 through 3 , has been known as standard induction therapy in AML in the past decades. Recently, the escalation of daunorubicin or cytarabine dose have shown benefit in the induction therapy. In adults under 60 years of age, previous trials have suggested that a daunorubicin dose of $90 \mathrm{mg}$ is superior to $45 \mathrm{mg}$, with the former showing an improved remission rate and survival benefit [11]. In older patients ( $>60$ years), a Korea trial showed a significant benefit of $90 \mathrm{mg} / \mathrm{m}^{2}$ both in remission rate and OS [12], which was particularly prominent in intermediate-risk patients in the ECOG1900 trial. In a randomized AML17 trail comparing $90 \mathrm{mg} / \mathrm{m}^{2}$ with $60 \mathrm{mg} / \mathrm{m}^{2}$, no significant difference was seen in remission rate or OS in any cytogenetic subgroup, with the 60-day mortality rate significantly higher in the high-dose (HD) daunorubicin group $\left(90 \mathrm{mg} / \mathrm{m}^{2}\right)(10 \mathrm{vs} 5 \%, P=0.001)$. However, it still remains necessary to take notice of molecular subgroups when it comes to longer follow-up, since in a recent E1900 trial with a median follow-up of 80 months, patients with Fms-like tyrosine kinase 3 (FLT3), nucleophosmin (NPM1), and DNA methyltransferase (DNMT)3A all benefited from HD daunorubicin [13].

In addition to daunorubicin, the administration of cytarabine at a daily dose of 100 to $200 \mathrm{mg} / \mathrm{m}^{2}$ for 7 to 10 days is also an important part in the standard induction therapy. In the EORTC-GIMEMA AML-12 trial with a median follow-up of 6 years in patients aged 15 to 60 years, higher remission and survival rate were observed in high-dose cytarabine $\left(3000 \mathrm{mg} / \mathrm{m}^{2}\right.$ per $12 \mathrm{~h}$ 
on days $1,3,5$, and 7) without significant toxicity. Particularly, for patients under 46 years old, the CR rate, event-free survival (EFS), and OS in high-dose cytarabine arm were significantly higher than standard dose cytarabine arm $\left(100 \mathrm{mg} / \mathrm{m}^{2}\right.$ per day continuously for 10 days) [14].

An alternative method to intensify standard induction regimen is the addition of a purine analog such as fludarabine or cladribine. One study demonstrated that the DAC regimen (DA plus cladribine), rather than DAF regimen (DA plus fludarabine), is associated with improved $\mathrm{OS}$ and $\mathrm{CR}$ in patients younger than 60 years with newly diagnosed AML. It is worth mentioning that both DAF and DAC increased CR rate in AML patients with adverse karyotype. Thus, further trials focusing on particular subgroups are needed [15]. Clofarabine is a second-generation purine analog, which has shown effectiveness in both R/R AML patients and newly diagnosed older patients $[16,17]$. A phase III study in newly diagnosed AML patients aged 18 to 65 years old confirmed the potent efficacy of clofarabine integrated in standard induction treatment, which showed reduced relapse probability without survival improvement. The study only found the survival benefit of clofarabine in subgroups of intermediate-risk AML and AML genotype without NPM1 and FLT3-ITD mutations [18].

\section{CPX-351}

CPX-351 is designed as a liposomal formulation of $7+3$ combination in a 5:1 ratio of cytarabine and daunorubicin, which was proved to be an optimal combination, with the highest level of synergy and the lowest level of antagonism [19, 20]. Two phase II randomized studies in 127 and 125 patients both confirmed a higher rate of CR (66.7 vs $51.2 \%$, and 49.3 vs $40.9 \%$, respectively) for patients treated with CPX-315 compared with those receiving $7+3$ regimen, and no difference in EFS or OS has been found in both phase II trials [21, 22]. However, it is worth mentioning that a phase III study, in which the studying group was not strictly limited, demonstrated an increased OS with daunorubicin in AML patients between the age of 60 and 65 years [23]. Another phase III study to confirm the efficacy of CPX-351 as first-line therapy in elderly patients (60-75 years) with high-risk (secondary) AML is ongoing (NCT01696084), which may make CPX-351 a better induction therapy for elderly patients who are not suitable for chemotherapy.

\section{Vosaroxin}

Vosaroxin is a quinolone derivative that intercalates DNA and inhibits topoisomerase II without producing oxygen free radicals, which has been confirmed to have better efficacy and lower cardiac toxicity than traditional anthracyclines [24]. Most recently, a large multicenter randomized phase III trial with 711 patients named VALOR demonstrated that the addition of vosaroxin to cytarabine resulted in a significant improvement in $\mathrm{CR}$ (30.1 vs $16.3 \%, P<0.0001)$ and OS (6.7 vs 5.3 months, $P$ $=0.024$ ) when censored for HSCT in R/R AML patients $\geq 60$ years [25]. With favorable efficacy and tolerability among older patients as well as notable survival benefits in subsets, vosaroxin stands a nice choice for novel combinatorial regimens, which will be further confirmed by future trials.

\section{Molecular targeted inhibitors Volasertib}

Volasertib (also known as BI 6727), which was awarded orphan drug status for AML in 2014, is a small-molecular inhibitor of polo-like kinases (PLKs), particularly PLK-1 (which was listed on Table 1). Inhibition of PLK1 overexpression in AML cell lines can bring about disorganized centrosome maturation, spindle assembly and cytokinesis during mitosis [20], and then cellular apoptosis subsequently. A phase II study made a comparison between the combination of volasertib with low-dose cytarabine (LDAC) and LDAC alone. The result confirmed greater clinical efficacy in the combination arm, statistically significant in $\mathrm{CR}$ (30 vs $13.3 \%, P=0.052$ ), median EFS (5.6 vs 2.3 months, $P$ $=0.021$ ), and median OS (8 vs 5.2 months, $P=0.047$ ) [26]. Meanwhile, there is also an ongoing phase III trial (NCT01721876) and a phase II trial of intensive chemotherapy with or without volasertib (NCT02198482).

\section{FLT3 inhibitors}

FLT3 is a class III tyrosine kinase receptor that stimulates normal hematopoiesis and cell proliferation in primitive hematopoietic stem and progenitor cells [27]. Although activating mutations in FLT3 are reported in only $30 \%$ of AML adults [28], FLT3 is constitutively expressed by autocrine signaling on leukemic cells in $70-100 \%$ of AML patients [29]. There are two types of FLT3 mutations, including approximately $20 \%$ of internal tandem duplications (FLT3/ITD) and $5 \sim 10 \%$ of point mutations in activating loop of tyrosine kinase domain (FLT3/TKD), constitutively activating cell proliferation and survival of leukemia blasts. Both mutations are associated with poor prognosis and outcome, particularly FLT3/ITD, with an estimation of 2-year diseasefree survival (DFS) rates of $20 \%$ and 4-year OS of $20 \%$ [30]. Of note, ITD mutations are associated with a poor prognosis due to a high relapse rate, and higher allelic ratios of mutated/wild-type variants confer a worse prognosis [31], suggesting a greater clinical response to selective FLT3-inhibitors [32]. Recent years have witnessed a growing development of several FLT3 inhibitors tested in clinical trials as either single agent or in combination with conventional chemotherapies, with the 
former usually associated with modest anti-tumor activity, transient reduction of blasts, and increased toxicity [33]. Though more tolerated than traditional cytotoxic agents, drug resistance has still posed a major challenge to patients treated with single FLT3 inhibitor, including F691, N676, and D835 mutation with kinase domain of FLT3-ITD [34].

\section{Sorafenib}

Sorafenib is a potent first-generation multikinase inhibitor with activity against FLT3/ITD receptor, which has been evaluated as either single agent [35-41] or in combination with chemotherapies [42-45]. SORAML is a placebo-controlled randomized study of adding sorafenib to daunorubicin and cytarabine $(7+3)$ in 267 newly diagnosed patients aged $18-60$ years. The addition of sorafenib resulted in a significantly prolonged 3-year EFS ( 40 vs $22 \%, P=0.013$ ) and RFS (56 vs $38 \%, P=0.017$ ) without improvement in OS and CR [46]. In contrast to this study, a second randomized study in 201 older patients aged 61-80 years showed no improvement in EFS, $\mathrm{CR}$, and OS, with a higher early mortality (17 versus $7 \%$, $P=0.052$ ) compared with placebo [44]. It can be seen from the difference of two studies that the combination of sorafenib with intensive chemotherapy may be too toxic for older patients, who have a poor prognosis mainly due to more resistance and less tolerance. Thus, for older patients, combining multikinase inhibitors with lower intensity therapies like hypomethylating agents (HMAs) may be an alternative choice [47]. Recent studies also suggest potential benefit of post-HSCT sorafenib in patients with FLT3-ITD [48].

\section{Midostaurin (PKC412)}

Midostaurin is a first-generation multi-target agent that inhibits FLT3, c-kit, platelet-derived growth factor receptor (PDGFR), vascular endothelial growth factor receptor (VEGFR), and protein kinase C [3]. As a well-tolerated and orally bioavailable agent, it enhances the response to induction chemotherapy and represents the potential to bridge mutant and wild-type (WT)-FLT3 AML patients to transplantation [49]. In two phase IIB studies of single-agent midostaurin administered in FLT3-mutated and FLT3-WT AML patients, there is a blast decrease $\geq$ $50 \%$ in the majority of $\mathrm{R} / \mathrm{R}$ or vulnerable/frail patients, especially those with FLT3 mutation, but CRs are rare and transient $[50,51]$. When it comes to combination, a phase IB trail adding midostaurin of two doses during (concomitant) or after (sequential) standard induction therapy confirmed a higher CR and lower toxicity in the lower-dose group (50 mg daily), as well as a higher CR rate in FLT3-mutated patients (92 vs 74\%) [52]. In the meantime, there was a multicenter, randomized phase III trial (RATIFY) in 717 younger adult patients, which demonstrated a significant improvement in OS and EFS among AML patients with FLT3 mutation, when adding midostaurin to standard induction therapy. In particular, the benefit of midostaurin was observed in patients undergoing transplantation during the first remission [53]. Thus, the combination regimen could be considered as first-line treatment in younger AML patient, while it is still uncertain whether the combination regimen might benefit older patients or those with wild-type FLT3. As is reported, combination with histone deacetylase (HDAC) inhibitors is also associated with a higher CR [54-56], and most recently, whether midostaurin improves RFS after transplant is under investigation (NCT01883362).

\section{Quizartinib (AC220)}

Quizartinib selectively inhibits FLT3/STK1, CSF1R/FMS, SCFR/KIT, and PDGFRs. A phase I trial in R/R AML patients determined the maximum tolerated dose (MTD) of $200 \mathrm{mg}$ per day with the dose-limiting toxicity (DLT) of grade 3 QTc prolongation [57]. Subsequently, several phase II trials studying with lower doses demonstrated prominent composite $\mathrm{CR}(\mathrm{CRc})$ rate ranging from 44 to $54 \%$ and ORR (CRc + PR) ranging from 61 to $72 \%$ in FLT3-ITD-positive patients [58-60]. Combination studies are ongoing (NCT01892371). As mentioned, single agent is proved to have limited efficacy due to drug resistance. Though active against FLT3-ITD mutation, most of tyrosine kinase inhibitors (TKIs) including quizartinib had no activity against FLT3-TKD mutation [34], the effect of which on the outcome remains unsettled.

\section{Crenolanib (CP868596)}

To maximize tolerability and response duration, novel FLT3 inhibitors like crenolanib, which is potent, selective, and invulnerable to resistance-conferring kinase domain mutation, are developed [61]. In addition to FLT3ITD mutation in nearly one third of AML patients [62], nowadays with the progress of more powerful FLT3 inhibitors being tested in many clinical trials, resistanceconferring point mutations like D835 and F691 have emerged during disease progression [63]. Crenolanib is a selective pan inhibitor active against both FLT3-ITD and FLT3-TKD D835 mutations, whereas most agents only have limited activity against the former. Crenolanib is a benzamidine quinolone derivative and currently a representative of the potent next-generation FLT3 TKIs.

\section{Gilteritinib (ASP2215)}

As potent as crenolanib, gilteritinib is also a selective next-generation FLT3 inhibitor with activity against both FLT3-ITD and FLT3-TKD mutations. A preclinical study compared gilteritinib with four other FLT3 inhibitors 
using immunoblotting and drew a conclusion that its in vitro efficacy is equal to or greater than the other TKIs (midostaurin, sorafenib, quizartinib, and crenolanib) and may be the most useful FLT3 inhibitor to date [64]. Worth mentioning, due to less activity against c-kit than quizartinib, gilteritinib has little myelosuppression.

\section{Lestaurtinib}

Lestaurtinib (CEP-701) is an orally bioavailable firstgeneration FLT3 inhibitor, as well as a potent inhibitor of JAK2 [65, 66]. Recently, a randomized assessment from UK AML 15 and AML 17 trials confirmed no statistically significant benefit observed in the combination of lestaurtinib with standard chemotherapy for newly diagnosed AML patients mostly younger than 60 years.

\section{NPM1 mutation}

NPM1 mutations represent the most frequent genetic alteration in AML, which are found in approximately $25 \%$ of patients with de novo AML. It is associated with improved outcomes, and the mechanisms have not been clearly elucidated. NPM1 is a promising therapeutic target for AML, since NPM1 mutations represent founder genetic lesions in leukemogenesis. Some recent studies have shown conflicting results on the association between NPM1 mutation and the response to ATRA or arsenic trioxide (ATO) adjunct to standard chemotherapy [67-69]. Interestingly, it has also been suggested that ATRA and arsenic trioxide combination can selectively induce apoptosis and differentiation in NPM1mutated cells, as well as promote leukemia regression in elderly patients unfit for induction chemotherapy [70, 71]. Furthermore, since NPM1-mutated leukemia cells are associated with increased CD33 expression [72], CD33 antibodies like gemtuzumab ozogamicin (GO) could be a targeted therapy for those NPM1-mutated patients with high CD33 expression. Finally, recent evidence has emerged that drugs such as dactinomycin, triggering a nucleolar stress response, may target NPM1-mutated AML [73].

\section{Epigenetic mutations and alterations}

Lately, epigenetic alterations that are heritable and reversible in contrast to genetic changes represent a focus of interest with respect to therapeutic targets in AML. With a rapid advance in all kinds of sequencing, recurrent mutated genes involved in epigenetic regulation have been identified, including TET2, IDH1, IDH2, DNMT3A, and EZH2. Abnormal DNA methylation and histone modification are two main modes of epigenetic dysregulation.

\section{DNMT inhibitors}

DNA methylation is catalyzed by DNMTs. Recurrent mutations in DNMT3A are found in 6 to $36 \%$ of AML patients, which is hypothesized to act as dominant negatives in leukemogenesis [74]. HMAs inhibiting DNMTs are options for older patients who cannot tolerate intensive chemotherapy with lower toxicities and equal efficacy. As known, azacitidine (5-Aza) and decitabine are two HMAs currently approved for clinical use, both of which have shown clinical benefit in clinical trials [75-78]. A phase II study of older patients who were unfit for intensive chemotherapy treated with 10-day schedule of decitabine yielded a CR rate of $47 \%$, without certain benefit observed in the combination of decitabine with HDAC valproic acid. Interestingly, patients harboring monosomy 7 or $\operatorname{del}(7 q)$ had a higher response rate of 91\% [79]. This study also proposed that higher pretreatment levels of miR-29b were associated with response $(P=0.02)$ to decitabine, allowing it to be a predictive marker and stratification tool in selection of older AML patients for this regimen. Further multicenter studied should be performed. Another single-institution trail suggested patients with unfavorable risk or TP53 mutations had significantly higher response rates to 10-day decitabine therapy despite their poor prognosis after cytotoxic chemotherapy [80]. In addition to this, the OS rate was similar among patients with unfavorable-risk and intermediate-risk cytogenetic profiles. It is worth mentioning that patients with TP53 mutations may not always be sensitive to single-agent decitabine treatment owing to the emergence of resistant subclones and incomplete mutation clearance. Still, decitabine should be considered as an important agent in the treatment of AML patients with TP53 mutations unfit for cytotoxic chemotherapy. To date, there are no therapies specifically targeting against DNMT3A.

Guadecitabine (SGI-110), as a second-generation HMA, is a dinucleotide of decitabine and deoxyguanosine resistant to cytidine deaminase and can prolong the exposure to decitabine in vivo. A phase I study assessed three treatment schedules of guadecitabine: daily schedule for 5 days continuously, weekly, and twice-weekly schedule. It was identified that the maximum demethylation was achieved with a dose of $60 \mathrm{mg} / \mathrm{m}^{2}$ per day for 5 days consecutively [81]. Likewise, a multicenter randomized phase I/II study accessing the safety and activity of two doses and schedules of guadecitabine in older AML patients also recommended the $60 \mathrm{mg} / \mathrm{m}^{2}$ guadecitabine in a 5-day regimen. A phase II study randomizing among 5-day regimen, 10-day regimen, and a combination of the 5-day schedule with idarubicin or cladribine is ongoing (NCT02096055), as well as a phase III study in progress to compare this 5-day schedule of 
guadecitabine with standard care. Also, another phase III randomized study of guadecitabine versus treatment choice in R/R AML has been initiated (NCT02920008). Anyway, therapeutic efficacy of guadecitabine will ultimately rely on a demonstrable improvement in OS; only then SGI-110 can be expected to become an alternative choice for patients ineligible for traditional induction chemotherapy due to old age, comorbidities, etc. [82].

Sapacitabine (CYC682) is a novel oral nucleoside analog. It is metabolized into the active metabolite CNDAC and incorporated into cellular DNA to exert anticancer activity by interfering with DNA synthesis and inducing cell cycle arrest. In a phase II trial, sapacitabine was administered to 60 AML patients aged 70 years or older from 12 centers in the USA, studying three dose schedules of sapacitabine: (A) $200 \mathrm{mg}$ bid for 7 days, (B) $300 \mathrm{mg}$ bid for 7 days, and (C) $400 \mathrm{mg}$ bid on days $1-3$ for 2 weeks. One-year OS was 35, 10, and 30\% in three groups, respectively. The 30 -day mortality was $13 \%$ and the 60-day mortality doubled [83]. In addition, SEAMLESS, a multicenter, randomized, phase III study of comparing sapacitabine alternating with decitabine to single agent decitabine in approximately 485 elderly patients aged 70 years or older, is ongoing (NCT01303796).

\section{IDH inhibitors}

IDH is one kind of enzyme that catalyzes the oxidative decarboxylation of isocitrate to alpha-ketoglutarate $(\alpha-K G)$, and the enzyme TET2 co-works with a-KG to convert 5methylcytosine $(5 \mathrm{mC})$ to 5 -hydroxymethylcytosine $(5 \mathrm{hmc})$, which promotes DNA demethylation. Mutant IDH $(\mathrm{mIDH})$ enzymes convert $\alpha-\mathrm{KG}$ to (R)-2-HG, which competitively inhibits a-KG-dependent enzymes including TET2. Besides, inactivating mutation in TET2 can lead to loss of function. Thus, both IDH and TET2 mutations can result in accumulation of 5mc and DNA hypermethylation and consequently promote AML.

Though prognostic impact of IDH gene mutations remains controversial, inhibitors targeted $\mathrm{mIDH}$ have been developed these days. Small-molecule mIDH inhibitors include AG-221, AG-120, AG-881, and IDH305, among which AG-221 and AG-120 have shown evidence of efficacy and are being tested in clinical trials. The first IDH2 inhibitor AG-221 is developed to inhibit mutant IDH2, reduce 2HG levels, and restore TET2 activity, thereby reversing $5 \mathrm{mC}$ accumulation in mouse $\mathrm{mIDH}$ AML models [84]. In ASH 2015, a phase I dose escalation and expansion study of AG-221 demonstrated an ORR of $41 \%$ and a true CR of $18 \%$ in patients with $R / R$ AML [85]. AG-120 monotherapy was associated with an ORR of $35 \%$ in a similar study [86]. Additionally, methylation inhibitors like 5-Aza can inhibit the conversion of cytosine to $5 \mathrm{mC}$ in TET2-mutant AML in mice, thereby preventing $5 \mathrm{mC}$ from accumulation. In a study, a comparison was drawn between the effects of preventing DNA hypermethylation induced by genetic loss of TET2 and restoring TET2 activity by inhibiting mutant IDH2 in AML [87]. Both AG-221 and 5-Aza induced differentiation of leukemic cells, but neither significantly killed mutant cells. Therefore, targeting epigenetic dysregulation could be an effective therapeutic strategy in AML, while dual-pronged therapies such as combining epigenetic inhibitors with kinase-targeted therapies may be a better choice [88]. Nowadays, both AG-120 and AG-221 are being investigated in newly diagnosed AML patients with IDH mutations, in combination with induction and consolidation chemotherapy (NCT02632708) and azacitidine (NCT02677922).

\section{HDAC inhibitors}

Histone modifications include acetylation and methylation which are reversibly mediated by histone acetyltransferases, HDACs, HMTs, and histone demethylases, respectively. Histone acetylation increases the accessibility of transcription factors to gene regions and consequently promotes gene expression. Conversely, deacetylation leads to transcriptional repression and impaired hematopoietic differentiation, which can be inhibited by HDAC inhibitors (HDACIs). When used as a single agent in MDS and AML, HDACIs seem to be modest [89]. Considering the disappointing results of combined clinical trials recently $[55,90-93]$, it remains a challenge to find an optimal combination regimen of HDACIs with other agents. Other histone modifiers like BET inhibitors (OTX015), DOT1L inhibitors (EPZ-2676), and LSD1 inhibitors (GSK2879552) are being investigated as monotherapy in clinical trials and still need further exploration.

\section{Immunotherapy for AML}

Cancer immunotherapy aims to stimulate the immune system to destroy tumors. Novel immunotherapies for AML mainly consist of monoclonal antibodies (mAbs), chimeric antigen receptor-engineered $\mathrm{T}$ cells (CAR $\mathrm{T}$ cells), and checkpoint inhibitors.

\section{Monoclonal antibodies}

Currently, the most encouraging therapeutic targets for AML are CD33 and CD123, which are both expressed in leukemic cells and normal hematopoietic cells. Due to off-tumor effects of aplasia and neutropenia, it is relatively more difficult to find an ideal target for AML than ALL. It was wildly known that GO, the first anti-CD33 mAb approved by the FDA in 2000, was withdrawn from market in 2010 due to early toxicity and little clinical benefit. Nevertheless, recent studies have demonstrated an improved survival in populations with favorable/ intermediate-risk cytogenetics [94-97]. Since older AML patients are not suited to cytotoxic chemotherapy, best 
supportive care (BSC) including hydroxyurea or lowdose cytarabine is considered despite dismal outcomes [98]. A randomized phase III EORTC-GIMEMA AML19 trial demonstrated a significant improved OS in older AML patients with single-agent low-dose GO $\left(6 \mathrm{mg} / \mathrm{m}^{2}\right.$ on day 1 and $3 \mathrm{mg} / \mathrm{m}^{2}$ on day 8), compared with BSC group (4.9 vs 3.6 months, $P=0.005$ ). Subgroup analysis confirmed the prediction that GO would be most effective in patients with high CD33 expression [99]. Phase IV clinical trials for patients with relapsed AML are now ongoing (NCT02312037). Given the above, GO monotherapy could embody a new choice for elderly patients. Besides, the combination of azacitidine and GO in phase II studies also revealed encouraging remission and survival rates in elderly patients $[100,101]$.

At ASH 2015, another CD33 antibody known as SGN$33 \mathrm{~A}$, presented promising results in a phase I study in combination with hypomethylating drugs in older patients [102]. These encouraging results have promoted the phase III CASCADE study (NCT019002329) which attempts to evaluate SGN-33A combined with 5-Aza or decitabine for older adults with newly diagnosed AML. In comparison with $\mathrm{GO}$, vadastuximab seems to have effective therapeutic results in poor-risk group in ongoing studies [103]. Other drugs like CSL362 and SL401 that target CD123 are now being investigated in various studies and have shown some promising data $[104,105]$.

\section{CAR T therapy}

Closely linked with graft-versus-host disease (GvHD), graft-versus-leukemic (GvL) effect appears after HSCT, via which the donor $\mathrm{T}$ cell plays an important role in killing leukemia cells. Elderly patients are not suitable candidates for HSCT due to high toxicity and relapse rate [106], and the efficacy of HSCT could be enhanced by infusion of CAR T cells [107]. CARs targeting CD19 have demonstrated remarkable potency in B cell malignancies such as B-ALL. The success of CD19 CAR T lies in two factors: (1) massive expansion and persistence of infused CAR T cells with costimulators and (2) tolerability of CD19 B cell aplasia due to its limited expression on mature B cells [108]. As mentioned, it remains challenging to find an ideal AML target owing to its profound and intolerable hematopoietic toxicity. Most of the current antigens in AML treatment are just overexpression antigens, rather than true AML-specific surface antigens, which brings about fatal off-tumor toxicity [109]. Several studies have proved in mouse models that targeting with anti-CD123 CAR T-cells (CD123 CART) and anti-CD33 CAR T-cells (CD33 CART) had some anti-AML potency but severe myeloablation was inevitable [110-112]. Particularly, a preclinical study in a mouse model using CD123 CART showed that CD123 expressed more frequently than CD33, and CD123 CART mediated potent in vivo antileukemic effect as well as increased survival of the majority of animals [110]. Furthermore, it is worth mentioning that the persistence of CAR $\mathrm{T}$ cells is associated with both anti-tumor efficacy and prolonged myeloablation, which suggests that a following rescue HSCT strategy is imperative. Future investigations in CAR T therapy warrant more focus on selection of specific AMLrelated surface targets.

\section{Checkpoint inhibitors}

In normal situations, immune checkpoints act as protective mechanism against autoimmunity, while tumor cells take advantage of them to evade immune system response and mediate immune resistance [113]. Thus, checkpoint inhibitors work via unleashing suppressed immune responses [114]. Two key checkpoint receptors are programmed cell death protein 1 (PD1) and cytotoxic Tlymphocyte-associated antigen 4 (CTLA4), both of which have been used in preclinical AML models [115]. One group treated three relapsed AML patients after alloHSCT with the PD-1 inhibitor nivolumab. Among these three patients, one achieved an ongoing CR, one experienced stabilization, and the third failed to respond. It suggested that targeting PD-1 might be an effective salvage therapy for relapsed AML after allo-HSCT, though the optimal dose of nivolumab to restore GvL effects without leading to severe GvHD still remains studying [116]. Phase II trials using single nivolumab or in combination with CTLA4 antibodies after allo-HSCT and chemotherapies are ongoing (NCT02532231, NCT02846376, NCT02464657).

\section{Conclusion}

The fundamental goal of precision medicine is to integrate population-based molecular, clinical, and other data to make individual-based clinical decisions for patients [117]. It has been demonstrated that the new formulated chemotherapies, molecular targeted agents, and immunotherapies all have clinical activity as single agents, but the activity seems limited. Recent studies have confirmed that combining with chemotherapy or other new drugs may bring more benefit for AML patients. The treatment of AML remains a tough challenge despite advances in our understanding of molecular mechanism and prognostic impact. Therefore, many questions are still unsolved in the use of these new drugs, which indicates that both patient and disease status should be taken into consideration. Massive efforts are required to pave the way for precision medicine in the foreseeable future. 


\section{Abbreviations}

5hmc: 5-Hydroxymethylcytosine; 5mC: 5-Methylcytosine; alloHSCT: Allogeneic HSCT; AML: Acute myeloid leukemia; APL: Acute promyelocytic leukemia; ATO: Arsenic trioxide; ATRA: All-transretinoic acid; CAR T cells: Chimeric antigen receptor-engineered T cells; CR: Complete remission; CTLA4: Cytotoxic T-lymphocyte-associated antigen 4; DLT: Doselimiting toxicity; DNMTs: DNA methyltransferases; EFS: Event-free survival; FLT3: Fms-like tyrosine kinase 3; GO: Gemtuzumab ozogamicin; GvHD: Graftversus-host disease; GVL: Graft-versus-leukemic; HDAC: Histone deacetylase; HMAs: Hypomethylating agents; HSCT: Hematopoietic stem cell transplantation; ITD: Internal tandem duplications; LDAC: Low-dose cytarabine; mAbs: Monoclonal antibodies; mIDH: Mutant IDH; MTD: Maximum tolerated dose; OS: Overall survival; PD1: Programmed cell death protein 1; PDGFR: Platelet-derived growth factor receptor; PLKs: Pololike kinases; R/R: Relapsed/refractory; TKD: Tyrosine kinase domain; TKIs: Tyrosine kinase inhibitors; VEGFR: Vascular endothelial growth factor receptor; WT: Wild type; a-KG: Alpha-ketoglutarate

\section{Acknowledgements}

None

\section{Funding}

This work was supported by the National Natural Science Foundation of China (81430004), Tianjin Clinical Research Center for Blood Diseases (15ZXLCSY00010), and CAMS Initiative Fund for Medical Sciences (2016-12M-1-001).

\section{Availability of data and materials}

Not applicable

\section{Authors' contributions}

XY drafted the manuscript; JW revised the manuscript. All authors read and approved the final manuscript.

\section{Ethics approval and consent to participate}

Not applicable

\section{Consent for publication}

Not applicable

\section{Competing interests}

The authors declare that they have no competing interests.

\section{Publisher's Note}

Springer Nature remains neutral with regard to jurisdictional claims in published maps and institutional affiliations.

Received: 22 September 2017 Accepted: 19 December 2017 Published online: 05 January 2018

\section{References}

1. Döhner H, Estey EH, Amadori S, Appelbaum FR, Büchner T, Burnett AK, et al. Diagnosis and management of acute myeloid leukemia in adults: recommendations from an international expert panel, on behalf of the European LeukemiaNet. Blood. 2010;115:453-74.

2. Döhner H, Weisdorf DJ, Bloomfield CD. Acute myeloid leukemia. N Engl J Med. 2015;373:1136-52.

3. Stein EM, Tallman MS. Emerging therapeutic drugs for AML. Blood. 2016; 127:71-8

4. Li S, Mason CE, Melnick A. Genetic and epigenetic heterogeneity in acute myeloid leukemia. Curr Opin Genet Dev. 2016;36:100-6.

5. Marcucci G, Haferlach T, Döhner H. Molecular genetics of adult acute myeloid leukemia: prognostic and therapeutic implications. J Clin Oncol. 2011;29:475-86

6. Zeijlemaker W, Gratama J-W, Schuurhuis G. Tumor heterogeneity makes AML a "moving target" for detection of residual disease. Cytometry B Clin Cytom. 2014;86:3-14.

7. McCulloch EA. Stem cells in normal and leukemic hemopoiesis (Henry Stratton Lecture). Blood. 1983;62:1-13.

8. Bonnet D. Normal and leukaemic stem cells. Br J Haematol. 2005:130: 469-79.
9. Ravandi F, Burnett AK, Agura ED, Kantarjian HM. Progress in the treatment of acute myeloid leukemia. Cancer. 2007;110:1900-10.

10. Kantarjian H, Ravandi F, O'Brien S, Cortes J, Faderl S, Garcia-Manero G, et al. Intensive chemotherapy does not benefit most older patients (age 70 years or older) with acute myeloid leukemia. Blood. 2010;116:4422-9.

11. Fernandez HF, Sun Z, Yao X, Litzow MR, Luger SM, Paietta EM, et al. Anthracycline dose intensification in acute myeloid leukemia. N Engl J Med. 2009;361:1249-59.

12. Lee J-H, Joo Y-D, Kim H, Bae SH, Kim MK, Zang DY, et al. A randomized trial comparing standard versus high-dose daunorubicin induction in patients with acute myeloid leukemia. Blood. 2011;118:3832-41.

13. Luskin MR, Lee JW, Fernandez HF, Abdel-Wahab O, Bennett JM, Ketterling $\mathrm{RP}$, et al. Benefit of high-dose daunorubicin in AML induction extends across cytogenetic and molecular groups. Blood. 2016;127:1551-8.

14. Willemze R, Suciu S, Meloni G, Labar B, Marie J-P, Halkes CJ, et al. High-dose cytarabine in induction treatment improves the outcome of adult patients younger than age 46 years with acute myeloid leukemia: results of the EORTC-GIMEMA AML-12 trial. J Clin Oncol. 2013;32:219-28.

15. Holowiecki J, Grosicki S, Giebel S, Robak T, Kyrcz-Krzemien S, Kuliczkowski K et al. Cladribine, but not fludarabine, added to daunorubicin and cytarabine during induction prolongs survival of patients with acute myeloid leukemia: a multicenter, randomized phase III study. J Clin Oncol. 2012;30:2441-8.

16. Kantarijian H, Gandhi V, Cortes J, Verstovsek S, Du M, Garcia-Manero G, et al. Phase 2 clinical and pharmacologic study of clofarabine in patients with refractory or relapsed acute leukemia. Blood. 2003;102:2379-86.

17. Burnett AK, Russell NH, Hunter AE, Milligan D, Knapper S, Wheatley K, et al. Clofarabine doubles the response rate in older patients with acute myeloid leukemia but does not improve survival. Blood. 2013;122:1384-94.

18. Löwenberg B, Pabst T, Maertens J, van Norden Y, Biemond BJ, Schouten HC et al. Therapeutic value of clofarabine in younger and middle aged (18-65 years) adults with newly diagnosed AML. Blood. 2017;129:1636-45.

19. Mayer LD, Harasym TO, Tardi PG, Harasym NL, Shew CR, Johnstone SA, et al. Ratiometric dosing of anticancer drug combinations: controlling drug ratios after systemic administration regulates therapeutic activity in tumor-bearing mice. Mol Cancer Ther. 2006;5:1854-63.

20. Giles FJ. New drugs in acute myeloid leukemia. Curr Oncol Rep. 2002:4: $369-74$

21. Lancet JE, Cortes JE, Hogge DE, Tallman MS, Kovacsovics TJ, Damon LE, et al. Phase 2 trial of CPX-351, a fixed 5: 1 molar ratio of cytarabine/ daunorubicin, vs cytarabine/daunorubicin in older adults with untreated AML. Blood. 2014;123:3239-46.

22. Cortes JE, Goldberg SL, Feldman EJ, Rizzeri DA, Hogge DE, Larson M, et al. Phase II, multicenter, randomized trial of CPX-351 (cytarabine: daunorubicin) liposome injection versus intensive salvage therapy in adults with first relapse AML. Cancer. 2015;121:234-42.

23. Löwenberg B, Ossenkoppele GJ, van Putten W, Schouten HC, Graux C, Ferrant A, et al. High-dose daunorubicin in older patients with acute myeloid leukemia. N Engl J Med. 2009;361:1235-48.

24. Stuart RK, Cripe LD, Maris MB, Cooper MA, Stone RM, Dakhil SR, et al. REVEAL-1, a phase 2 dose regimen optimization study of vosaroxin in older poor-risk patients with previously untreated acute myeloid leukaemia. Br J Haematol. 2015;168:796-805

25. Ravandi F, Ritchie EK, Sayar H, Lancet JE, Craig MD, Vey N, et al. Vosaroxin plus cytarabine versus placebo plus cytarabine in patients with first relapsed or refractory acute myeloid leukaemia (VALOR): a randomised, controlled, double-blind, multinational, phase 3 study. Lancet Oncol. 2015;16:1025-36.

26. Döhner $\mathrm{H}$, Lübbert $\mathrm{M}$, Fiedler $\mathrm{W}$, Fouillard $\mathrm{L}$, Haaland $\mathrm{A}$, Brandwein JM, et al. Randomized, phase 2 trial of low-dose cytarabine with or without volasertib in AML patients not suitable for induction therapy. Blood. 2014:124:1426-33

27. Ferrara F. New agents for acute myeloid leukemia: is it time for targeted therapies? Expert Opin Investig Drugs. 2012;21:179-89.

28. Daver $\mathrm{N}$, Konopleva M. Sorafenib and novel multikinase inhibitors in AML. Lancet Oncol. 2015:16:1582-3.

29. Thiede C, Steudel C, Mohr B, Schaich M, Schäkel U, Platzbecker U, et al. Analysis of FLT3-activating mutations in 979 patients with acute myelogenous leukemia: association with FAB subtypes and identification of subgroups with poor prognosis. Blood. 2002;99:4326-35.

30. Marcucci G, Maharry K, Whitman SP, Vukosavljevic T, Paschka P, Langer C, et al. High expression levels of the ETS-related gene, ERG, predict adverse outcome and improve molecular risk-based classification of cytogenetically 
normal acute myeloid leukemia: a Cancer and Leukemia Group B Study. J Clin Oncol. 2007;25:3337-43.

31. Borthakur G, Kantarjian H, Patel KP, Ravandi F, Qiao W, Faderl S, et al. Impact of numerical variation in FMS-like tyrosine kinase receptor 3 internal tandem duplications on clinical outcome in normal karyotype acute myelogenous leukemia. Cancer. 2012;118:5819-22.

32. Pratz KW, Sato T, Murphy KM, Stine A, Rajkhowa T, Levis M. FLT3-mutant allelic burden and clinical status are predictive of response to FLT3 inhibitors in AML. Blood. 2010;115:1425-32.

33. Sudhindra A, Smith CC. FLT3 inhibitors in AML: are we there yet? Curr Hematol Malig Rep. 2014;9:174-85.

34. Smith CC, Wang Q, Chin C-S, Salerno S, Damon LE, Levis MJ, et al. Validation of ITD mutations in FLT3 as a therapeutic target in human acute myeloid leukaemia. Nature. 2012;485:260-3.

35. Borthakur G, Kantarjian H, Ravandi F, Zhang W, Konopleva M, Wright JJ, et al. Phase 1 study of sorafenib in patients with refractory or relapsed acute leukemias. Haematologica. 2011;96:62-8.

36. Lee SH, Paietta E, Racevskis J, Wiernik PH. Complete resolution of leukemia cutis with sorafenib in an acute myeloid leukemia patient with FLT3-ITD mutation. Am J Hematol. 2009;84:701-2.

37. Metzelder SK, Wollmer E, Neubauer A, Burchert A. Sorafenib in relapsed and refractory FLT3-ITD positive acute myeloid leukemia: a novel treatment option. Dtsch Med Wochenschr. 2010;135:1852-6.

38. Mohan BP, How G, Loh Y, Linn YC. Sorafenib monotherapy gives sustainable suppression of FLT3 clone in untreated patients with FLT3internal tandem duplication positive acute myeloid Leukaemia. $\mathrm{Br} J$ Haematol. 2012;157:131-2.

39. Mori M, Sprague J. The successful remission induction by sorafenib and long-term complete remission in a FLT3-ITD-positive patient with a refractory acute erythroid leukemia and abnormal cytogenetics. Leuk Res. 2012;36:e1-3.

40. Safaian N, Czibere A, Bruns I, Fenk R, Reinecke P, Dienst A, et al. Sorafenib (Nexavar ${ }^{\circ}$ induces molecular remission and regression of extramedullary disease in a patient with FLT3-ITD+ acute myeloid leukemia. Leuk Res. 2009; 33:348-50.

41. Schroeder T, Zohren F, Saure C, Bruns I, Czibere A, Safaian NN, et al. Sorafenib treatment in 13 patients with acute myeloid leukemia and activating FLT3 mutations in combination with chemotherapy or as monotherapy. Acta Haematol. 2010;124:153-9.

42. Ravandi F, Yi CA, Cortes JE, Levis M, Faderl S, Garcia-Manero G, et al. Final report of phase II study of sorafenib, cytarabine, and idarubicin for initial therapy in younger patients with acute myeloid leukemia. Leukemia. 2014; 28:1543.

43. Zhang QY, Wei XD, Yin QS, Mi RH, Yuan FF, Chen L. Sorafenib in combination with chemotherapy as first-line therapy for FLT3-ITD positive acute myeloid leukemia. Zhonghua Xue Ye Xue Za Zhi. 2017;38:415-20.

44. Serve H, Krug U, Wagner R, Sauerland MC, Heinecke A, Brunnberg U, et al. Sorafenib in combination with intensive chemotherapy in elderly patients with acute myeloid leukemia: results from a randomized, placebo-controlled trial. J Clin Oncol. 2013;31:3110-8.

45. Uy GL, Mandrekar SJ, Laumann K, Marcucci G, Zhao W, Levis MJ, et al. A phase 2 study incorporating sorafenib into the chemotherapy for older adults with FLT3-mutated acute myeloid leukemia: CALGB 11001. Blood Adv. 2017;1:331-40.

46. Röllig C, Serve H, Hüttmann A, Noppeney R, Müller-Tidow C, Krug U, et al. Addition of sorafenib versus placebo to standard therapy in patients aged 60 years or younger with newly diagnosed acute myeloid leukaemia (SORAML): a multicentre, phase 2, randomised controlled trial. Lancet Oncol. 2015;16:1691-9.

47. Ravandi F, Alattar ML, Grunwald MR, Rudek MA, Rajkhowa T, Richie MA, et al. Phase 2 study of azacytidine plus sorafenib in patients with acute myeloid leukemia and FLT-3 internal tandem duplication mutation. Blood. 2013;121:4655-62.

48. Brunner AM, Li S, Fathi AT, Wadleigh M, Ho VT, Collier K, et al. Haematopoietic cell transplantation with and without sorafenib maintenance for patients with FLT3-ITD acute myeloid leukaemia in first complete remission. Br J Haematol. 2016;175:496-504.

49. Gallogly MM, Lazarus HM. Midostaurin: an emerging treatment for acute myeloid leukemia patients. J Blood Med. 2016;7:73-83.

50. Stone RM, DeAngelo DJ, Klimek V, Galinsky I, Estey E, Nimer SD, et al. Patients with acute myeloid leukemia and an activating mutation in FLT3 respond to a small-molecule FLT3 tyrosine kinase inhibitor, PKC412. Blood. 2005;105:54-60.

51. Fischer T, Stone RM, DeAngelo DJ, Galinsky I, Estey E, Lanza C, et al. Phase IIB trial of oral midostaurin (PKC412), the FMS-like tyrosine kinase 3 receptor (FLT3) and multi-targeted kinase inhibitor, in patients with acute myeloid leukemia and high-risk myelodysplastic syndrome with either wild-type or mutated FLT3. J Clin Oncol. 2010;28:4339-45.

52. Stone RM, Fischer T, Paquette R, Schiller G, Schiffer CA, Ehninger G, et al. Phase IB study of the FLT3 kinase inhibitor midostaurin with chemotherapy in younger newly diagnosed adult patients with acute myeloid leukemia. Leukemia. 2012;26:2061-8.

53. Stone RM, Mandrekar SJ, Sanford BL, Laumann K, Geyer S, Bloomfield CD, et al. Midostaurin plus chemotherapy for acute myeloid leukemia with a FLT3 mutation. N Engl J Med. 2017;377:454-64.

54. Cooper BW, Kindwall-Keller TL, Craig MD, Creger RJ, Hamadani M, William WT, et al. A phase I study of midostaurin and azacitidine in relapsed and elderly AML patients. Clin Lymphoma Myeloma Leuk. 2015;15:428-32. e422.

55. Strati P, Kantarjian H, Ravandi F, Nazha A, Borthakur G, Daver N, et al. Phase I/II trial of the combination of midostaurin (PKC412) and 5-azacytidine for patients with acute myeloid leukemia and myelodysplastic syndrome. Am J Hematol. 2015;90:276-81.

56. Williams CB, Kambhampati S, Fiskus W, Wick J, Dutreix C, Ganguly S, et al. Preclinical and phase I results of decitabine in combination with midostaurin (PKC412) for newly diagnosed elderly or relapsed/refractory adult patients with acute myeloid leukemia. Pharmacotherapy. 2013;33: 1341-52.

57. Levis M. Quizartinib for the treatment of FLT3/ITD acute myeloid leukemia. Future Oncol. 2014;10:1571-9.

58. Cortes JE, Perl AE, Dombret H, Kayser S, Steffen B, Rousselot P, et al. Final results of a phase 2 open-label, monotherapy efficacy and safety study of quizartinib (AC220) in patients $\geq 60$ years of age with FLT3 ITD positive or negative relapsed/refractory acute myeloid leukemia(ASH annual meeting abstracts). Blood. 2012;120:Abstract 48.

59. Levis MJ, Perl AE, Dombret H, Döhner H, Steffen B, Rousselot P, et al. Final results of a phase 2 open-label, monotherapy efficacy and safety study of quizartinib (AC220) in patients with FLT3-ITD positive or negative relapsed/ refractory acute myeloid leukemia after second-line chemotherapy or hematopoietic stem cell transplantation(ASH annual meeting abstracts). Blood. 2012:120:Abstract 673.

60. Cortes JE, Tallman MS, Schiller G, Trone D, Gammon G, Goldberg S, et al. Results of a phase 2 randomized, open-label, study of lower doses of quizartinib (AC220; ASP2689) in subjects with FLT3-ITD positive relapsed or refractory acute myeloid leukemia (AML) (ASH annual meeting abstracts). Blood. 2013:122:Abstract 494

61. Smith CC, Lasater EA, Lin KC, Wang Q, McCreery MQ, Stewart WK, et al. Crenolanib is a selective type I pan-FLT3 inhibitor. Proc Natl Acad Sci. 2014; 111:5319-24.

62. Levis M, Small D. FLT3: ITDoes matter in leukemia. Leukemia. 2003;17: 1738-52.

63. Galanis A, Ma H, Rajkhowa T, Ramachandran A, Small D, Cortes J, et al. Crenolanib is a potent inhibitor of FLT3 with activity against resistanceconferring point mutants. Blood. 2014;123:94-100.

64. Lee LY, Hernandez D, Rajkhowa T, Smith SC, Raman JR, Nguyen B, et al. Preclinical studies of gilteritinib, a next-generation FLT3 inhibitor. Blood. 2017;129:257-60.

65. Hexner EO, Serdikoff C, Jan M, Swider CR, Robinson C, Yang S, et al. Lestaurtinib (CEP701) is a JAK2 inhibitor that suppresses JAK2/STAT5 signaling and the proliferation of primary erythroid cells from patients with myeloproliferative disorders. Blood. 2008;111:5663-71.

66. Hexner EO, Mascarenhas J, Prchal J, Roboz GJ, Baer MR, Ritchie EK, et al. Phase I dose escalation study of lestaurtinib in patients with myelofibrosis. Leukemia Lymphoma. 2015;56:2543-51.

67. Schlenk RF, Lübbert M, Benner A, Lamparter A, Krauter J, Herr W, et al. Alltrans retinoic acid as adjunct to intensive treatment in younger adult patients with acute myeloid leukemia: results of the randomized AMLSG 0704 study. Ann Hematol. 2016;95:1931-42.

68. Nazha A, Bueso-Ramos C, Estey E, Faderl S, O'Brien S, Fernandez MH, et al. The addition of all-trans retinoic acid to chemotherapy may not improve the outcome of patient with NPM1 mutated acute myeloid leukemia. Front Oncol. 2013;3:218 
69. Burnett AK, Hills RK, Green C, Jenkinson S, Koo K, Patel Y, et al. The impact on outcome of the addition of all-trans retinoic acid to intensive chemotherapy in younger patients with nonacute promyelocytic acute myeloid leukemia: overall results and results in genotypic subgroups defined by mutations in NPM1, FLT3, and CEBPA. Blood. 2010;115:948-56.

70. El Hajj H, Dassouki Z, Berthier C, Raffoux E, Ades L, Legrand O, et al. Retinoic acid and arsenic trioxide trigger degradation of mutated NPM1, resulting in apoptosis of AML cells. Blood. 2015;125:3447-54.

71. Martelli MP, Gionfriddo I, Mezzasoma F, Milano F, Pierangeli S, Mulas F, et al. Arsenic trioxide and all-trans retinoic acid target NPM1 mutant oncoprotein levels and induce apoptosis in NPM1-mutated AML cells. Blood. 2015;125: 3455-65.

72. De Propris MS, Raponi S, Diverio D, Milani ML, Meloni G, Falini B, et al. High CD33 expression levels in acute myeloid leukemia cells carrying the nucleophosmin (NPM1) mutation. Haematologica. 2011;96:1548-51.

73. Falini B, Brunetti L, Martelli MP. Dactinomycin in NPM1-mutated acute myeloid leukemia. N Engl J Med. 2015;373:1180-2.

74. Celik H, Mallaney C, Kothari A, Ostrander EL, Eultgen E, Martens A, et al. Enforced differentiation of Dnmt3a-null bone marrow leads to failure with c-Kit mutations driving leukemic transformation. Blood. 2015;125:619-28.

75. Fenaux P, Mufti GJ, Hellström-Lindberg E, Santini V, Gattermann N, Germing $U$, et al. Azacitidine prolongs overall survival compared with conventional care regimens in elderly patients with low bone marrow blast count acute myeloid leukemia. J Clin Oncol. 2009;28:562-9.

76. Issa J-PJ, Garcia-Manero G, Giles FJ, Mannari R, Thomas D, Faderl S, et al. Phase 1 study of low-dose prolonged exposure schedules of the hypomethylating agent 5-aza-2'-deoxycytidine (decitabine) in hematopoietic malignancies. Blood. 2004;103:1635-40.

77. Blum W, Klisovic RB, Hackanson B, Liu Z, Liu S, Devine H, et al. Phase I study of decitabine alone or in combination with valproic acid in acute myeloid leukemia. J Clin Oncol. 2007;25:3884-91.

78. Cashen AF, Schiller GJ, O'Donnell MR, DiPersio JF. Multicenter, phase II study of decitabine for the first-line treatment of older patients with acute myeloid leukemia. J Clin Oncol. 2009;28:556-61.

79. Blum W, Garzon R, Klisovic RB, Schwind S, Walker A, Geyer S, et al. Clinical response and miR-29b predictive significance in older AML patients treated with a 10-day schedule of decitabine. Proc Natl Acad Sci. 2010;107:7473-8.

80. Welch JS, Petti AA, Miller CA, Fronick CC, O'Laughlin M, Fulton RS, et al. TP53 and decitabine in acute myeloid leukemia and myelodysplastic syndromes. N Engl J Med. 2016;375:2023-36.

81. Issa J-PJ, Roboz G, Rizzieri D, Jabbour E, Stock W, O'Connell C, et al. Safety and tolerability of guadecitabine (SGI-110) in patients with myelodysplastic syndrome and acute myeloid leukaemia: a multicentre, randomised, doseescalation phase 1 study. Lancet Oncol. 2015;16:1099-110.

82. Kharfan-Dabaja MA. Guadecitabine for AML and MDS: hype or hope? Lancet Oncol. 2015;16:1009-11.

83. Kantarjian H, Faderl S, Garcia-Manero G, Luger S, Venugopal P, Maness $L$, et al. Oral sapacitabine for the treatment of acute myeloid leukaemia in elderly patients: a randomised phase 2 study. Lancet Oncol. 2012;13: 1096-104.

84. Yen K, Travins J, Wang F, David MD, Artin E, Straley K, et al. AG-221, a firstin-class therapy targeting acute myeloid leukemia harboring oncogenic IDH2 mutations. Cancer Discovery. 2017;7:478-93.

85. Metzeler KH, Herold T, Rothenberg-Thurley M, Amler S, Sauerland MC, Goerlich D, et al. Spectrum and prognostic relevance of driver gene mutations in acute myeloid leukemia. Blood. 2016;128:686-98.

86. Fan B, Le K, Manyak E, Liu H, Prahl M, Bowden CJ, et al. Longitudinal pharmacokinetic/pharmacodynamic profile of AG-120, a potent inhibitor of the IDH1 mutant protein, in a phase 1 study of IDH1-mutant advanced hematologic malignancies (ASH annual meeting abstracts). Blood. 2015;126: Abstract 1310.

87. Shih AH, Meydan C, Shank K, Garrett-Bakelman FE, Ward PS, Intlekofer AM, et al. Combination targeted therapy to disrupt aberrant oncogenic signaling and reverse epigenetic dysfunction in IDH2-and TET2-mutant acute myeloid leukemia. Cancer Discovery. 2017;7:494-505.

88. Losman JA. Cancer therapy: the leukaemia epigenome targeted. Nature. 2017;543:634-5.

89. Garcia-Manero G, Tambaro FP, Bekele NB, Yang H, Ravandi F, Jabbour E, et al. Phase II trial of vorinostat with idarubicin and cytarabine for patients with newly diagnosed acute myelogenous leukemia or myelodysplastic syndrome. J Clin Oncol. 2012;30:2204-10.
90. Lübbert M, Kuendgen A. Combining DNA methyltransferase and histone deacetylase inhibition to treat acute myeloid leukemia/myelodysplastic syndrome: achievements and challenges. Cancer. 2015;121:498-501.

91. Kirschbaum M, Gojo I, Goldberg SL, Bredeson C, Kujawski LA, Yang A, et al. A phase 1 clinical trial of vorinostat in combination with decitabine in patients with acute myeloid leukaemia or myelodysplastic syndrome. $\mathrm{Br} \mathrm{J}$ Haematol. 2014;167:185-93.

92. Issa JP, Garcia-Manero G, Huang X, Cortes J, Ravandi F, Jabbour E, et al. Results of phase 2 randomized study of low-dose decitabine with or without valproic acid in patients with myelodysplastic syndrome and acute myelogenous leukemia. Cancer. 2015;121:556-61.

93. Prebet T, Sun Z, Figueroa ME, Ketterling R, Melnick A, Greenberg PL, et al. Prolonged administration of azacitidine with or without entinostat for myelodysplastic syndrome and acute myeloid leukemia with myelodysplasia-related changes: results of the US leukemia intergroup trial E1905. J Clin Oncol. 2014;32:1242-8.

94. Petersdorf SH, Kopecky KJ, Slovak M, Willman C, Nevill T, Brandwein J, et al. A phase 3 study of gemtuzumab ozogamicin during induction and postconsolidation therapy in younger patients with acute myeloid leukemia. Blood. 2013;121:4854-60

95. Burnett AK, Hills RK, Milligan D, Kjeldsen L, Kell J, Russell NH, et al. Identification of patients with acute myeloblastic leukemia who benefit from the addition of gemtuzumab ozogamicin: results of the MRC AML15 trial. J Clin Oncol. 2010;29:369-77.

96. Lichtenegger FS, Krupka C, Köhnke T, Subklewe M. Immunotherapy for acute myeloid leukemia. Semin Hematol. 2015;52:207-14.

97. Hills RK, Castaigne S, Appelbaum FR, Delaunay J, Petersdorf S, Othus M, et al. Addition of gemtuzumab ozogamicin to induction chemotherapy in adult patients with acute myeloid leukaemia: a meta-analysis of individual patient data from randomised controlled trials. Lancet Oncol. 2014;15:986-96.

98. Oran B, Weisdorf DJ. Survival for older patients with acute myeloid leukemia: a population-based study. Haematologica. 2012;97:1916-24.

99. Walter RB, Raden BW, Kamikura DM, Cooper JA, Bernstein ID. Influence of CD33 expression levels and ITIM-dependent internalization on gemtuzumab ozogamicin-induced cytotoxicity. Blood. 2005;105:1295-302.

100. Nand S, Othus M, Godwin JE, Willman CL, Norwood TH, Howard DS, et al. A phase 2 trial of azacitidine and gemtuzumab ozogamicin therapy in older patients with acute myeloid leukemia. Blood. 2013;122:3432-9.

101. Kurimoto M, Matsuoka H, Hanaoka N, Uneda S, Murayama T, Sonoki T, et al. Pretreatment of leukemic cells with low-dose decitabine markedly enhances the cytotoxicity of gemtuzumab ozogamicin. Leukemia. 2013;27:233-5.

102. Fathi AT, Erba HP, Lancet JE, Stein EM, Walter RB, DeAngelo DJ, et al. SGNCD33A plus hypomethylating agents: a novel, well-tolerated regimen with high remission rate in frontline unfit AML (ASH annual meeting abstracts). Blood. 2015;126:Abstract 454.

103. Bixby DL, Stein AS, Fathi AT, Kovacsovics TJ, Levy MY, Erba HP, et al. Vadastuximab talirine monotherapy in older patients with treatment naive CD33-positive acute myeloid leukemia (AML) (ASH annual meeting abstracts). Blood. 2016;128:Abstract 590.

104. Frankel AE, Konopleva M, Hogge D, Rizzieri D, Brooks C, Cirrito T, et al. Activity and tolerability of SL-401, a targeted therapy directed to the interleukin-3 receptor on cancer stem cells and tumor bulk, as a single agent in patients with advanced hematologic malignancies. J Clin Oncol. 2013;31:7029.

105. Lee EM, Yee D, Busfield SJ, McManus JF, Cummings N, Vairo G, et al. Efficacy of an Fc-modified anti-CD123 antibody (CSL362) combined with chemotherapy in xenograft models of acute myelogenous leukemia in immunodeficient mice. Haematologica. 2015;100:914-26.

106. Duval M, Klein JP, He W, Cahn J-Y, Cairo M, Camitta BM, et al. Hematopoietic stem-cell transplantation for acute leukemia in relapse or primary induction failure. J Clin Oncol. 2010;28:3730-8.

107. Barrett DM, Singh N, Porter DL, Grupp SA, June CH. Chimeric antigen receptor therapy for cancer. Annu Rev Med. 2014;65:333-47.

108. Gill S. Chimeric antigen receptor T cell therapy in AML: how close are we? Best Pract Res Clin Haematol. 2016;29:329-33.

109. Fan M, Li M, Gao L, Geng S, Wang J, Wang Y, et al. Chimeric antigen receptors for adoptive T cell therapy in acute myeloid leukemia. J Hematol Oncol. 2017;10:151.

110. Gill S, Tasian SK, Ruella M, Shestova O, Li Y, Porter DL, et al. Preclinical targeting of human acute myeloid leukemia and myeloablation using chimeric antigen receptor-modified T cells. Blood. 2014;123:2343-54. 
111. Kenderian S, Ruella M, Shestova O, Klichinsky M, Aikawa V, Morrissette J, et al. CD33-specific chimeric antigen receptor T cells exhibit potent preclinical activity against human acute myeloid leukemia. Leukemia. 2015;29:1637-47.

112. Pizzitola I, Anjos-Afonso F, Rouault-Pierre K, Lassailly F, Tettamanti S, Spinelli $\mathrm{O}$, et al. Chimeric antigen receptors against CD33/CD123 antigens efficiently target primary acute myeloid leukemia cells in vivo. Leukemia. 2014;28: 1596-605.

113. Pardoll DM. The blockade of immune checkpoints in cancer immunotherapy. Nat Rev Cancer. 2012;12:252-64.

114. Topalian SL, Drake CG, Pardoll DM. Immune checkpoint blockade: a common denominator approach to cancer therapy. Cancer Cell. 2015;27: 450-61

115. Zhang L, Gajewski TF, Kline J. PD-1/PD-L1 interactions inhibit antitumor immune responses in a murine acute myeloid leukemia model. Blood. 2009; 114:1545-52.

116. Albring J, Inselmann S, Sauer T, Schliemann C, Altvater B, Kailayangiri S, et al. PD-1 checkpoint blockade in patients with relapsed AML after allogeneic stem cell transplantation. Bone Marrow Transplant. 2017;52:317-20.

117. Biankin AV. The road to precision oncology. Nat Genet. 2017:49:320-1.

Submit your next manuscript to BioMed Central and we will help you at every step:

- We accept pre-submission inquiries

- Our selector tool helps you to find the most relevant journal

- We provide round the clock customer support

- Convenient online submission

- Thorough peer review

- Inclusion in PubMed and all major indexing services

- Maximum visibility for your research

Submit your manuscript at www.biomedcentral.com/submit
Biomed Central 